\title{
Persuasion Processes in Consumer Intent to Read Online Product Reviews: A Study Based on the Elaboration Likelihood Model
}

\author{
Meng-Lin Shih, *Shu-Hui Chuang
}

Department of Information Technology, Ching Kuo Institute of Management and Health, Fu Hsin Rd., Keelung 20301, Taiwan

\author{
mlshih@mis.ccu.edu.tw
}

*Department of Business Administration, Asia University, Liufeng Rd., Wufeng, Taichung, Taiwan 41354

joyce@asia.edu.tw

\section{ABSTRACT}

Studies have shown that many prospective consumers have the intention of reading online reviews of a product before purchasing that product online. How this intention arises, however, has not been extensively investigated. The study described here used the elaboration likelihood model (ELM) to examine the central and peripheral persuasion processes involved in shaping consumers' intent to read online product reviews. These two processes were operationalized by respectively using perceived review quality and perceived review consistency as constructs in a model of persuasion to read online reviews. The findings of the study suggest that consumers' intent to read online product reviews is shaped by their perception of such reviews as being either of high quality or as consistent with their prior knowledge about the product being reviewed. For an individual consumer, the persuasiveness of one of these processes over the other depends on the two respective moderators of consumer involvement and consumer expertise.

Keywords: Consumer, elaboration likelihood model (ELM), online product purchasing, online product review

\section{Council for Innovative Research}

Peer Review Research Publishing System

Journal: INTERNATIONAL JOURNAL OF COMPUTERS \& TECHNOLOGY

Vol 12, No. 10

editor@cirworld.com

www.cirworld.com, www.ijctonline.com 


\section{INTRODUCTION}

In the past two decades, the mode of shopping for merchandise has shifted dramatically from buying during a physical visit to a retail store to reviewing and purchasing merchandise through an online retail outlet in the worldwide web. Shopping at online retail outlets is particularly strong during holiday seasons, and contributes substantially to overall retail sales at these times, demonstrating the economic importance of online shopping. In contrast to shopping via physical visits to retail stores, however, shopping at online retail outlets cannot employ the five human neural senses in selecting merchandise for purchase, obligating the consumer to instead base their purchasing decisions on physically limited representations of a product such as photographs and text descriptions presented at a website. Given this situation, online product reviews appear to be exceptionally important to consumers, who seem likely to regard these reviews, which convey the reactions of other consumers of a product, as evidence of the intrinsic nature and value of the product (Burnkrant \& Cousineau, 1975; Chen et al., 2012; Poulos \& Korfiatis, 2013).

Online product reviews are new forms of communication that function as recommendations for purchasing or not purchasing a product (Park et al., 2007; Park \& Kim, 2008; Sparks et al., 2013). They resemble traditional oral evaluations in acting as communications of other consumers' opinions about products (Chatterjee, 2001) Unlike such oral evaluations, however, online product reviews express the views of persons unknown to the viewer, and therefore carry less credibility for the viewer than do direct consumer opinions from family members or friends (Ratchford et al., 2001; Xie et al., 2011). Despite this, there is compelling evidence that many consumers have the intent to read online reviews of a product before shopping online for that product. Exemplifying this is that half of consumers who visit online shopping malls consider it important to read product reviews in making their buying decisions (Piller, 1991). In a survey of 1000 individuals shopping online for components of a vacation, $53 \%$ stated that apart from price, other persons' reviews were the most important factor in their choice of vacation destinations and accommodations (Walsh, 2007). The reason for the willingness of so many consumers to rely on others' online reviews must be that most consumers have already been persuaded by such reviews and must have thus formed the intent to refer to them when shopping online. Unfortunately, neither the factors responsible for the formation of this intent nor those that persuade online consumers to buy a product have been defined.

The study reported here was conducted to examine the processes responsible for consumers' intent to read online product reviews. It used the elaboration likelihood model (ELM) (Eagly \& Chaiken, 1993; Petty \& Cacioppo, 1986) of persuasion to identify these processes, to discern which of these process is most effective in persuading consumers to seek online reviews, and to identify any factors that modify these processes. Although the study is still new and continuing, it may help the understanding of consumers' motivation to read online product reviews.

\section{THEORY AND HYPOTHESES}

The social psychology literature has described the use of the ELM in determining the role of persuasive processes in shaping a targeted human behaviour (Petty \& Cacioppo, 1986; Petty et al., 1981; SanJosé-Cabezudo et al., 2009). The ELM is founded on the concept that two routes of persuasion, a central route and a peripheral route, which differ in the degree of reflective information processing they involve, govern the formation of individual perceptions. The central route requires an individual to think carefully about issue-related arguments in an informational message, and to scrutinize the relative merits and relevance of those arguments before establishing a perception that contributes to a target behaviour. The peripheral route of persuasion is more likely to be used in processing simple cues that induce the formation of a perception without 
requiring reflection on the rational merits of the information being presented. This two-route concept has been applied in the context of usage of information technology (IT) (Bhattacherjee \& Sanford, 2006), where it has shown that both the content and sources of information about a particular IT are equally effective in shaping users' attitudes toward using that IT. This means that an individual user may be persuaded to accept an IT by experts' opinions about it (peripheral cues), although such acceptance may sometimes rely on the quality of the experts' arguments in terms of their rationale or the evidence they provide (argument quality). On the premise that online consumers are also and simultaneously users of IT (Koufaris, 2002), the ELM appears to be as readily applicable to investigating their online information-seeking and purchasing behaviour toward merchandise presented via IT as toward IT itself.

\subsection{Effects of perceived review quality and consistency on intent to read online reviews}

The postulate for the study was that consumers' intent to read online reviews of a product is founded in the central and peripheral routes of persuasion. Typically, research based on the ELM operationalizes "argument quality," or the persuasive strength of arguments embedded in an informational message, as the key element or construct in persuasion through the central route (Lee \& Xia, 2011). However, the present study relies on "perceived review quality," or the quality of the contents of a review from the perspective of their informational characteristics, as the key element or variable in persuasion through the central route. This is based on the demonstrably persuasive effect of this variable on consumers' intent to buy a certain product found in other studies of central-route processing based on the ELM (Park et al., 2007). Furthermore, in contrast to classical ELM-based research, which uses "source credibility," or the extent to which users perceive an information source as believable, competent, and trustworthy, as a peripheral cue in persuasion (Petty et al., 1981), the present study utilizes "perceived review consistency," or the extent to which a review is consistent with the consumer's prior knowledge about a product (Zhang \& Watts, 2004), as a peripheral cue. A similar construct, called "information consistency," has also been considered a peripheral cue and has demonstrated its persuasive power among users of online communications (Zhang \& Watts, 2004). In short, the perceived quality and consistency of an online product review, both of which are pivotal in shaping a consumer's intent to read other online reviews, are used as the respective central- and peripheral-route constructs in the present study. Accordingly, it was hypothesized that:

H1: Perceived review quality positively affects consumers' intent to read online product reviews.

H2: Perceived review consistency positively affects consumers' intent to read online product reviews.

\subsection{Moderators of persuasion processes}

Individuals choose one of the two routes of persuasion set forth in the ELM on the basis of their ability and motivation to process arguments, which in the ELM fall into the category known as likelihood of elaboration (Petty \& Cacioppo, 1986). Individuals with a high likelihood of elaboration are energized, ready, and willing to engage in careful scrutiny or thoughtful processing of an informational message, and therefore tend to be more persuaded by the quality of an argument than by peripheral cues. Contrastingly, individuals with a low likelihood of elaboration lack the motivation or ability to deliberate thoughtfully, and are more likely to be persuaded by peripheral cues than by the quality of an argument. In short, individual motivation and ability are presumed to moderate the effects of argument quality and peripheral cues on a target behaviour (Maclnnis et al., 1991). Drawing on research with the ELM, the present study operationalized the motivational dimension of the likelihood of elaboration as consumer involvement, defined as a consumer's ability to become involved with the 
information presented in a product review, and operationalized the ability aspect of the likelihood of elaboration as consumer expertise, defined as the ability to read an online product review.

Consumers deeply involved in the process of reading an online product review engage in processing the information it contains through the central route, by focusing on content cues such as the perceived quality of the review. Conversely, consumers with a low level of involvement in the review will be less likely to engage in elaboration and more likely to be affected by cues processed via the peripheral route of persuasion, such as the perceived consistency of the review. Therefore, two further hypotheses for the present study were that:

H3: The greater the individual involvement in the topic of an online review, the more the perceived quality of the review will affect the individual's intent to read online product reviews.

H4: The greater the individual involvement in the topic of an online review, the less the perceived consistency of the review will affect the intent to read online product reviews.

Beyond this, the greater the individual level of expertise in the topic of an online product review, the more important will be its argument-relevant informational content in persuading the reader to use online reviews, and the greater will be the ability of the individual to understand that content. This in turn increases the likelihood of central-route processing and reliance on cues such as perceived review quality, and reduces the likelihood of reliance on peripheral cues such as review consistency (Ratneshwar \& Chaiken, 1991). Therefore:

H5: The greater the individual expertise in the topic of an online review, the more will perceived review quality affect the individual's intent to read online product reviews.

H6: The greater the individual expertise in the topic of an online review, the less will the perceived review consistency affect the individual's intent to read online product reviews.

\section{MATERIALS AND METHODS}

\subsection{Questionnaire development}

The questionnaire used in the study was developed to quantify the following five constructs of interest in the study: (1) intent to read online reviews, (2) perceived review quality, (3) perceived review consistency, (4) consumer involvement in the topics of online reviews, and (6) consumer expertise in the topics of online reviews. All five constructs were quantified on the basis of items validated in prior research, which were reworded to relate specifically to the context of reading online product reviews (see Appendix A). The construct for the intent to read reviews was adapted from Ajzen and Fishbein (1980) and Taylor and Todd (1995), and was modified to suit the context of online product reviews. The construct designated as perceived review quality, derived from a five-item measurement drawn from Park et al. (2007). focuses on the relevance, objectivity, understandability, and adequacy of explanation on online product reviews. Perceived review consistency was assessed with a three-item instrument adapted from an information-consistency scale developed by Zhang and Watts (2004) on the basis of a study by Vandenbosch and Higgins (1996). The quantification of consumer involvement and expertise was based on the original scales for these two measures that were developed and validated by Stamm and Dube (1994).

Before conducting the main survey, we conducted a pretest and a pilot test to validate the questionnaire used as the survey 
instrument from which the constructs used in the study were derived. In the pre-test, the instruments, scales, and questions used in the survey were reviewed by 10 faculty members of the Department of Information Management. Each of these faculty members has expertise in an area of research corresponding to one or more of the constructs used in the study. The pretest phase of the study increased the validity of the items used in developing the questionnaire used as the survey instrument, and improved the wording of the instructions, scales, and questions used in this instrument. The purpose of the pilot-test phase of the study was to test the reliability of the survey instrument and to identify ambiguities, unclear questions, and poorly worded questions in the instrument. The instrument was then tested by convenience sampling, which yielded 50 responses, of which 38 were complete, giving a valid response rate of $76 \%$. The results of the pilot test of the survey instrument were evaluated with Cronbach's test for the reliability of scales. Cronbach's coefficient of reliability, $\alpha$, was first calculated for the items used in developing each of the five constructs used in the study. The standard lower boundary for Cronbach's $\alpha$ is 0.70 (Hair et al., 1998).

\subsection{Data collection}

The research model for the study was tested with data from reviewers on the Mobile01 (www.mobile01.com) website. This website was chosen because it is an important online discussion site for consumer electronics, computer, and communications (3C) products in Taiwan. The reviewers were students at Asia University in Taiwan, who were recruited by a research assistant in the study and were regarded as appropriate surrogates for online shoppers because they had most of the attributes expected of this latter population (Torkzadeh \& Dhillon, 2002). The students' participation was voluntary, and each participant received NT\$100 to compensate for the time spent in reading reviews on the Mobile01website and filling out the survey questionnaire used in the study. The participants were instructed to answer all of the survey questions on the basis of their experience in reading product reviews on the Mobile01 website.

The survey yielded 265 responses, which were screened for usability and reliability, and of which 16 were eliminated as having come from respondents who were obviously unconcerned about their responses (e.g., those giving the same rating for all items in the questionnaire), leaving 249 questionnaires that were considered suitable for data analysis and from which the pretest data were derived. Of the survey respondents, approximately, 54.8\% were male; $59.1 \%$ were between 20 and 39 years of age; $93.5 \%$ were educated to the level of a college degree or above; and $50 \%$ used the Internet for more than 18 hours each week. Table 1 presents the descriptive statistics for the main constructs used in the study, including their means and standard deviations. Because the responses to the survey questionnaire were derived through a nonrandom method of sampling, a test of homogeneity on the survey respondents' demographic variables. All items in the five study constructs were tested against demographic control variables (gender, age, level of education, and Internet usage) through analysis of variance (ANOVA), as suggested by Cho (2006). The mean scores of all of the items tested were nonsignificantly $(P>0.05)$ represented by the demographic control variables.

\section{RESULTS}

The study data were analyzed with the partial least squares (PLS) method, a technique of structural equation modeling that has become widely accepted because of its accuracy and utility. The PLS method also puts minimal restrictions on sample size and residual distribution. The data analysis was done in two stages. In the first stage, all of the measurement scales used in the study were tested for reliability and construct validity using confirmatory factor analysis (CFA). In the second stage of the analysis, the structural model for the study was analyzed. The two-stage model was selected in preference to an 
alternative one-stage approach in which measurement scales and structural models are examined simultaneously, because the two-stage approach provides a more complete and robust test of measurement validity by examining potential associations between constructs that may not otherwise be theoretically specified in the one-stage structural model.

Table 1. Descriptive Statistics

\begin{tabular}{lcc}
\hline \hline Construct & Mean & SD \\
\hline Perceived review quality & 5.140 & 0.762 \\
Perceived review consistency & 5.025 & 0.873 \\
Intention of reading reviews & 5.797 & 1.008 \\
Consumer involvement & 4.797 & 1.038 \\
Consumer expertise & 4.795 & 1.034 \\
\hline \hline
\end{tabular}

\subsection{Validation of measurement scales}

Table 2 shows the standardized loading and other metrics for the item measures used in developing the study constructs, as well as measures of scale reliability and validity. Hair et al. (1998) suggested that in a sample of 150 respondents, a factor loading of 0.45 or greater is significant. In our study, all items in the measurement model exhibited factor loadings ranging from 0.711 to 0.962 and were therefore considered acceptable for the remainder of the analysis. The reliability metrics for all of the five constructs used as the independent variables in the study model, ranging from 0.865 to 0.962 , exceeded the recommended threshold of 0.70 for Cronbach's $\alpha$ (Segars, 1997), and were therefore also fully acceptable. Calculation of the average variance extracted (AVE) showed that eight AVE values exceeded the recommended threshold of 0.50 (Segars, 1997).

Table 3 shows the discriminant validity of the measurements used in the study. For good discriminant validity, the square root of the AVE of a construct should exceed that of the construct's correlations with the other constructs in a structural model (Fornell \& Larcker, 1987). The data in the study indicated that the shared variance among variables was smaller than the variances extracted for the constructs, which were the values for the diagonals connecting the constructs, or independent variables in the study model, with the main outcome measure, or dependent variable, of intent to read online reviews. This shows that the constructs were empirically distinct.

\subsection{Testing of hypotheses}

We used PLS with the bootstrapping resampling procedure to test our model. The examination of a structural equation model includes examination of the coefficients of the causal relationships between the constructs in the model, which would validate the hypothesized effects of the constructs, and the values of $r^{2}$, which indicate the variance in dependent variables explained by their antecedents. The main effects model in our study, $41.8 \%$ of the variance in the dependent or outcome variable of consumers' intent to read online product reviews was explained by perceived review quality $(\beta=0.412, P<$ $0.001)$ and perceived review consistency $(\beta=0.254, P<0.001)$, respectively supporting hypotheses 1 and 2 in the study, presented earlier. 
The product indicator approach proposed by Chin et al. (2003) was used to measure the model of moderating effects on the study constructs. This model tested the extent to which consumer involvement and consumer expertise, representing elaboration motivation and ability, respectively, moderated the main effects comprised in hypotheses 1 and 2 . An examination of individual routes in the moderating-effects model revealed that consumer involvement had a significantly

Table 2. Data Assessment for the Measurement Model Used in the Study

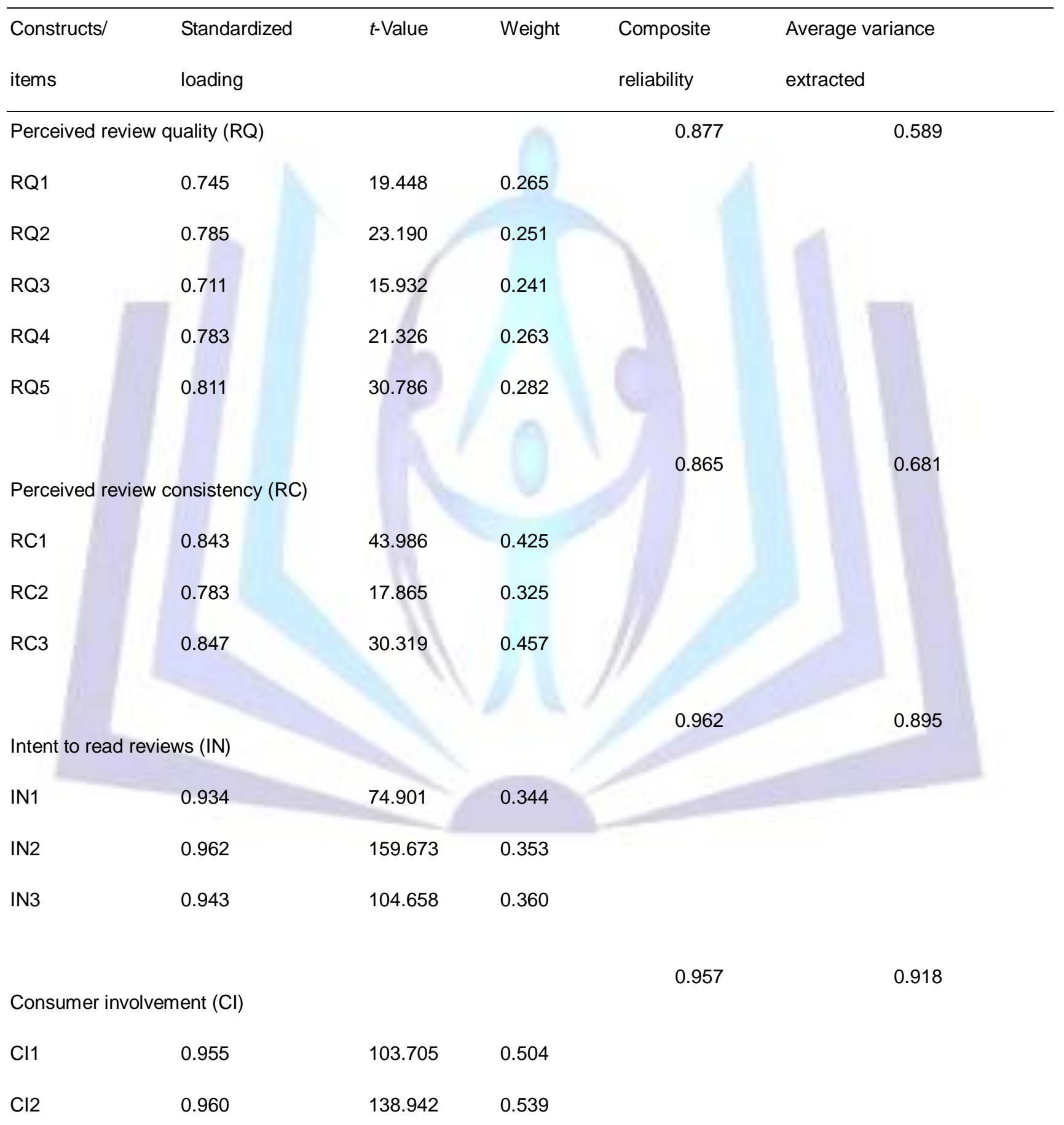


Table 3. Discriminant Validity

\begin{tabular}{|c|c|c|c|c|c|}
\hline Constructs & $\mathrm{RQ}$ & $\mathrm{RC}$ & IN & $\mathrm{Cl}$ & CE \\
\hline $\mathrm{RQ}$ & 0.590 & & & & \\
\hline $\mathrm{RC}$ & 0.373 & 0.681 & & & \\
\hline IN & 0.506 & 0.402 & 0.895 & & \\
\hline $\mathrm{Cl}$ & 0.298 & 0.158 & 0.254 & 0.918 & \\
\hline CE & 0.297 & 0.157 & 0.253 & 0.582 & 0.697 \\
\hline
\end{tabular}

positive moderating effect on the association between perceived review quality and the intent to read online product reviews $(\beta=0.169, P<0.01)$, as posited in hypothesis 3 . Conversely, consumer involvement negatively influenced the effect of perceived review consistency on the intent to read product reviews $(\beta=-0.096, P<0.05$ ), as posited in hypothesis 4. Consumer expertise positively moderated the effect of perceived review quality on the intent to read online product reviews $(\beta=0.156, P<0.01$ ), in accord with hypothesis 5 , and negatively moderated the effects of perceived review consistency on the intent to read such reviews $(\beta=-0.111, P<0.05)$, in accord with hypothesis 6 . Hypotheses 3 and 4 repeat this theoretical argument for the moderators of involvement, since involvement also increases the likelihood of elaboration. Hypotheses 5 and 6 posit that expertise will moderate the effects of perceived review quality and perceived review consistency on the intent to read online product reviews. Our model suggests that for individuals with high levels of expertise, the quality of online product reviews will strongly affect the intent to read such reviews, whereas for those with lower levels of expertise, cuses relating to consistency will have a stronger effect.

\section{DISCUSSION}

At the beginning of this report, we described three major points relating to the understanding of consumer intent to reading online product reviews. The first was that a consumer's perceptions from reading reviews at a retail website determine whether the consumer maintains an intention to read reviews for each purchase made at the website. A consumer who perceives that most online product reviews at a website are of high quality and consistent with the consumer's prior knowledge about the reviewed products will be more favorably disposed to reading other reviews posted at the website. This is consistent with the postulate in the ELM that central-route (e.g., perceived review quality) and peripheral-route (e.g., 
perceived review consistency) variables are equally important in shaping a consumer's behaviour. The second finding, based on the ELM literature, is that the central and peripheral routes can be operationalized, as was done in the present study through the use of constructs based on perceived review quality and perceived review consistency, respectively, and through the capture of elaboration motivation and ability with constructs based on consumer involvement and consumer expertise, respectively. Although consumer involvement positively moderated the central-route persuasion process involving perceived review quality, and negatively moderated the peripheral-route process involving perceived review consistency, as theoretically expected, consumer expertise had a positively moderating effect on central-route processes and a negatively moderating effect on peripheral-route processes. The analysis of the study data resolved this potential anomaly by revealing that while perceived review consistency was a salient peripheral cue for consumers with low elaboration, it was also a review-relevant quality for consumers with high elaboration. Combining these two groups of consumers into a single group and testing the resulting collective moderating influence on the effect of perceived review consistency masked the differential nature of the effects of the two elaboration states. The third point raised in the opening discussion was that the present study is as important for electronic retailers as other, prior research in this area. However, unlike prior research, which was primarily based on study of the ways in which positive and negative online reviews respectively helped or hindered online product purchases, the present study examined the factors that motivate consumers to read online product reviews, with the goal of identifying consumers' views of such product reviews themselves. The hypothesis behind this was that before shopping online, consumers will read reviews that are logical, persuasive, and provide sufficient rationale for buying a product on the basis of specific facts about the product. Conversely, consumers will not read online reviews that are abusively negative, emotional, or deficient in the quality of the reasoning used to support their statements, in the form of reasons based on facts about a product. The basis for the study was that such evidence can help online vendors to decide whether or not to add an online discussion forum to their websites, and how to properly manage the product reviews for such a forum if they decide to do this. Even though some online vendors may hesitate to adopt the strategy of providing a venue at which consumers can voice their opinions, from fear of product defamation through such a venue, they ought nevertheless to take into account the product reviews provided by third parties (e.g., at eopinion.com, or www.mobile01.com), because reviews of high quality that are consonant with consumers' prior experience with products discussed at these online for a strongly influence consumers' purchasing decisions.

\section{CONCLUSIONS}

The findings in the present study show that both the central and peripheral routes of persuasion are viable pathways through which to persuade consumers to read online reviews. In the central route, consumers engage in reflective processing of issue-related arguments in an informational message, whereas in the peripheral route they simply attend to cues about the consistency of an online product review. These mechanisms of behavioural influence shape consumers' intent to read online product reviews. The results of the present study confirm that both of these routes of influence are moderated by consumers' involvement and expertise in processing issue-relevant arguments. Consumers with higher elaboration involvement and expertise tend to be more influenced by the central route of persuasion, whereas those with lower involvement and expertise are more influenced by the peripheral route.

Although this study offers important insights into an unexplored topic, it is not without limitations. The study surveyed the views or perceptions that consumers kept in mind after reading product reviews at a functioning website 
(www.mobile01.com), and examined these consumers' willingness to refer to related product reviews at that same website if buying a product online. The study data showed that the website used in the study is not the online retail outlet most frequently used by our sample population, but does permit online shopping as well as providing a forum for consumer discussion. In Taiwan, the most commonly utilized consumer websites include Yahoo.com, PChome.com, and ETMall.com. A more influential study, of greater significance, would use product reviews at these online outlets for a model of the effect of online product reviews on consumer behaviour. Although this deficiency may have hurt the internal validity of our findings, the field-based data collection done in the present study improves the external validity or generalizability of the study findings as compared to that of studies conducted in laboratory settings, as have been most of the studies so far conducted of consumers' online retail behaviour. We urge that future studies use a controlled experimental design to verify the internal validity of the results of the present study.

\section{REFERENCES}

[1] Ajzen, I. \& Fishbein, M. (1980). Understanding Attitudes and Predicting Social Behavior. Prentice-Hall, Englewood Cliffs, NJ.

[2] Bhattacherjee, A. \& Sanford, C. (2006). Influence processes for information technology acceptance: An elaboration likelihood model. MIS Quarterly , 30(4), 805-825.

[3] Burnkrant, R.E. \& Cousineau, A. (1975). Informational and normative social influence in buyer behavior. Journal of Consumer Research, 2(3), 206-214.

[4] Chatterjee, P. (2001). Online reviews: Do consumers use them? Advances in Consumer Research, 28, $129-133$.

[5] Chen, L., Qi, L., \& Wang, F. (2012). Comparison of feature-level learning methods for mining online consumer reviews. Expert System with Applications, 39(10), 9588-9601.

[6] Chin, W.W., Marcolin, B. \& Newsted, P. (2003). A partial least squares latent variable modeling approach for measuring interaction effects: results form a Monte Carlo simulation study and an electronic-mail emotion/adoption study. Information Systems Research, 14(2), 189-217.

[7] Cho, V. (2006). A study of the roles of trusts and risks in information-oriented online legal services using an integrated model. Information \& Management, 43(4), 502-520.

[8] Eagly, A.H. \& Chaiken, S. (1993). The Psychology of Attitudes. Harcourt Brace Jovanovich, Fort Worth, TX.

[9] Fornell, C. \& Larcker, D. (1987). A second generation of multivariate analysis: classification of methods and implications for marketing research. In Houston MJ (ed.): Review of Marketing. American Marketing Association, Chicago, 407-450.

[10] Hair, J.F., Anderson, R.E., Tatham, R.L. \& Black, W.C. (1998). Multivariate Data Analysis with Readings, ed. 5. Prentice-Hall, Englewood Cliffs, NJ.

[11] Korfiatis, N., \& Poulos, M. (2013). Using online consumer reviews as a source for demographic recommendations: A case study using online travel reviews. Expert Systems with Applications, 40(14), 5507-5515.

[12] Koufaris, M. (2002). Applying the technology acceptance model and flow theory to online consumer behavior. Information Systems Research, 13(2), 205-223.

[13] Lee, G. \& Xia, W. (2011). A longitudinal experimental study on the interaction effects of persuasion quality, user training, and first-hand use on user perceptions of new information technology. Information \& Management, 48(7), 288-295.

[14] Maclnnis, D.J., Moorman, C. \& Jaworski, B.J. (1991). Enhancing and measuring consumers' motivation, opportunity, and ability to process brand information from ads. Journal of Marketing, 55(4), 32-53. 
[15] Park, D. \& Kim, S. (2008). The effects of consumer knowledge on message processing of electronic word-of-mouth via online consumer reviews. Electronic Commerce Research and Application, 7, 399-410.

[16] Park, D., Lee, J. \& Han, I. (2007). The effect of on-line consumer reviews on consumer purchasing intention: The moderating role of involvement. International Journal of Electronic Commerce, 11(4), 125-148.

[17] Petty, R.E. \& Cacioppo, J.T. (1986). Communication and Persuasion: Central and Peripheral Routes to Attitude Change. Springer-Verlag, New York.

[18] Petty, R.E., Cacioppo, J.T. \& Goldman, R. (1981). Personal involvement as a determinant of argument-based persuasion. Journal of Personality and Social Psychology, 41(5), 847-855.

[19] Piller, C. (1991). Everyone Is a Critic in Cyberspace. Los Angeles Times, December 3, A1.

[20] Ratchford, B.T., Talukdar, D. \& Lee, M. (2001). A model of consumer choice of the Internet as an information source. International Journal of Electronic Commerce, 5(3), 7-21.

[21] Ratneshwar, S. \& Chaiken, S. (1991). Comprehension's role in persuasion: The case of its moderating effect on the persuasive impact of source cues. Journal of Consumer Research, 18, 52-62.

[22] SanJosé-Cabezudo, R., Gutiérrez-Arranz, A.M. \& Gutiérrez-Cillán, J. (2009). The combined influence of central and peripheral routes in the online persuasion process. CyberPsychology \& Behavior, 12, 299-307.

[23] Segars, A.H. (1997). Assessing the unidimensionality of measurement: a paradigm and illustration within the context of information systems research. Omega, 25, 107-122.

[24] Sparks, B. A., Perkins, H. E., \& Buckley, R. (2013). Online travel reviews as persuasive communication: The effects of content type, source, and certification logos on consumer behavior. Tourism Management, 39 (December), 1-9.

[25] Stamm, K. \& Dube, R. (1994). The relationship of attitudinal components to trust in media. Communication Research, 21(1), 105-123.

[26] Taylor, S. \& Todd, P. (1995). Understanding information technology usage: A test of competing models. Information Systems Research, 6(2), 144-176.

[27] Torkzadeh, G. \& Dhillon, G. (2002). Measuring factors that influence the success of Internet commerce. Information Systems Research, 13, 187-204.

[28] Vandenbosch, B. \& Higgins, C. (1996). Information acquisition and mental models: An investigation into the relationship between behavior and learning. Information Systems Research, 7, 198-214.

[29] Walsh, C. (2007). Online Reviews Drive Net Sales. Travel Trade Gazette UK \& Ireland, 2, 24.

[30] Xie, H., Miao, Li, Kuo, P. \& Lee, B. (2011). Consumers' responses to ambivalent online hotel reviews: The role of perceived source credibility and pre-decisional disposition. International Journal of Hospitality Management, 30(1), 178-183.

[31] Zhang, W. \& Watts, S. (2004). Knowledge adoption in online communities of practice. Systems d'Information et Management, 9, 81-102. 


\section{Appendix A. Quantitative Measures Used in the Study Constructs}

Perceived review quality $(\mathrm{RQ})$

RQ1. Most reviews I read have sufficient reasons supporting the opinions.

RQ2. Most reviews I read are objective.

RQ3. Most reviews I read are understandable.

RQ4. Most reviews I read are credible.

RQ5. In general, the quality of most reviews I read is high.

Intent to read online reviews (IN)

IN1. I plan to read this website's product reviews when I buy a product online.

IN2. I intend to read this website's product reviews when I buy a product online.

IN3. I expect to read this website's product reviews when I buy a product online.

\section{Perceived review consistency $(\mathrm{RC})$}

RC1. The reviews I read reinforced my confidence in my future purchasing plan.

RC2. The arguments in the reviews I read are consistent with my existing knowledge about the product I want to buy.

RC3. The reviews I read verified my assumptions for my future purchasing plan.

\section{Consumer involvement $(\mathrm{Cl})$}

Cl1. How involved are you on the topic of the online reviews you read? (Not at all...a great deal)

Cl2. How much has the issue discussed in the online reviews been on your mind lately? (Not at all...a great deal)

\section{Consumer expertise (CE)}

CE1. How informed are you on the subject matter of the online reviews you read? (Novice...expert)

CE2. To what extent are you an expert on the topics of the online reviews you read? (Not at all...to a great extent) 